Intermetallics. Vol. 15. Núm. 2. 2007. Pag. 193-200

http://dx.doi.org/10.1016/j.intermet.2006.05.007

\title{
Structural ordering and magnetic properties of arc-melted FeGa alloys
}

J.M. Borrego, J.S. Blázquez, C.F. Conde and A. Conde

Departamento de Física de la Materia Condensada, Instituto de Ciencia de Materiales, C.S.I.C., Universidad de Sevilla, P.O. Box 1065, 41080 Sevilla, Spain

S. Roth

Leibniz Institut für Metallische Werkstoffe, IFW Dresden, Postfach 270016, D-01171 Dresden, Germany

\begin{abstract}
X-ray diffraction, Mössbauer spectrometry and magnetic properties have been performed on FeGa arc-melted binary alloys in order to study the compositional dependence of the structural ordering and magnetic properties of these alloys. The average magnetic hyperfine field at $300 \mathrm{~K}$ decreases with increasing Ga content as does the average magnetic moment per Fe atom and a linear dependence between both quantities is found for Ga content up to 20 at. \%. The substitution of Fe by Ga atoms increases the lattice parameter and causes a change in the order of the crystal structure that has been modelled using a binomial distribution method. The effect of Ga atoms as near neighbours of Fe on the average magnetic hyperfine field is evaluated.
\end{abstract}

Keywords: A-Magnetic intermetallics, B-Magnetic properties, B-Order/disorder transformations, F-Mössbauer spectroscopy 
Intermetallics. Vol. 15. Núm. 2. 2007. Pag. 193-200

http://dx.doi.org/10.1016/j.intermet.2006.05.007

\section{Introduction}

FeGa alloys have attracted great attention in the last few years due to their superior magnetostrictive properties at low saturation field [1-4]. This quality associated with their high mechanical strengh, good ductility, negligible magnetic hysteresis and low cost [5] makes these materials very promising for sensor and actuator applications.

Recent investigations include the study of the influence of the thermal history and the structural ordering on their magnetostriction [6,7], the phase equilibria and stability of ordered bcc phases in the Fe-rich portion of the FeGa system [8] and the structural transformations in quenched FeGa alloys [9]. Moreover, recently it was found that the addition of $\mathrm{Cu}$ to $\mathrm{Fe}_{65.5} \mathrm{Cr}_{4} \mathrm{Mo}_{4} \mathrm{Ga}_{4} \mathrm{P}_{12} \mathrm{C}_{5} \mathrm{~B}_{5.5}$ amorphous alloy produces a nanocrystalline microstructure consistent of FeGa nanocrystals embedded in an amorphous matrix [10]. These nanocrystalline alloys are interesting since they would combine a large glassforming ability with good soft magnetic properties.

However, a Mössbauer study of the FeGa alloy series is still missing. The importance of this study is double. On one hand, structure determination of FeGa alloys by X-ray diffraction is troublesome due to the similarity in the atomic scattering factors of $\mathrm{Fe}$ and $\mathrm{Ga}$ atoms which results in superlattice reflections of very low intensity. For example, the intensity of the strongest superlattice reflection associated with $\mathrm{DO}_{3}$ longrange order is calculated to be only $0.6 \%$ of the strongest primary reflection. Mössbauer spectrometry, as a short range order sensitive technique, does allow distinguishing between ordered and disordered atomic structures. For nanocrystalline alloys and due to the complexity of their magnetic hyperfine structure, the characterization of the hyperfine parameters of FeGa crystals becomes an essential information.

In the present work, arc-melted polycrystalline $\mathrm{Fe}_{100-\mathrm{y}} \mathrm{Ga}_{\mathrm{y}}(\mathrm{y}=5,10,20$ and 25$)$ alloys were prepared in order to study variations in the atomic short range order at room 
Intermetallics. Vol. 15. Núm. 2. 2007. Pag. 193-200

http://dx.doi.org/10.1016/j.intermet.2006.05.007

temperature with substitution of Fe by Ga. A local model based on a binomial distribution method [11] was used in order to correlate the magnetic hyperfine parameters with the structural ordering of these alloys. The influence of Ga substitution for Fe on the lattice parameter, saturation magnetization, magnetic moment and Curie temperature of these alloys is also reported. The interest of this study is focused in demonstrating that Mössbauer technique is able to supply detailed quantitative data for the structural ordering of such systems as Fe-Ga binary alloy where conventional X-ray diffraction techniques can not resolve an ordered structure due to the similarity between the scattering factors of its constituents.

\section{Experimental}

$\mathrm{Fe}_{100-\mathrm{y}} \mathrm{Ga}_{\mathrm{y}}(\mathrm{y}=5,10,20$ and 25 at. \%) alloys were prepared by arc-melting from pure $\mathrm{Fe}(99.95 \%)$ and $\mathrm{Ga}(99.999 \%)$ in an Edmund-Bühler high vacuum arc-melting system. The arc-melted bottoms $(\sim 2 \mathrm{~cm}$ diameter and $\sim 0.8 \mathrm{~mm}$ thick $)$ were remelted several times to get homogeneous samples.

$\mathrm{X}$-ray diffraction (XRD) patterns were recorded at room temperature using a Brucker AXS D8-Advance diffractometer with $\mathrm{Cu} \mathrm{K}_{\alpha}$ radiation on slices $\sim 100 \mu \mathrm{m}$ thick cut from the bulk samples.

For thermomagnetic (TMG) experiments, the magnetic field of a small magnet $(\sim 20 \mathrm{mT})$ was applied to the sample and the temperature variation of the magnetic force was recorded in a thermobalance (Perkin-Elmer TGA-7) as an apparent weight change of the sample. The Curie temperature, $T_{C}$, was determined from low field magnetization curves, by the "kink point" method. 
Intermetallics. Vol. 15. Núm. 2. 2007. Pag. 193-200

http://dx.doi.org/10.1016/j.intermet.2006.05.007

The saturation magnetization, $M_{s}$, was measured on samples $\sim 5 \mathrm{mg}$ at room temperature with a Lakeshore 7407 vibrating sample magnetometer (VSM), using a maximum applied field of $15 \mathrm{kOe}$.

Mössbauer spectra on the same samples studied by XRD were taken at $300 \mathrm{~K}$ in a transmission geometry using a ${ }^{57} \mathrm{Co}(\mathrm{Rh})$ source, with the $\gamma$-beam perpendicular to the ribbon plane. The values of the hyperfine parameters were refined using NORMOS [12] and MOSFIT programs [13]. The isomer shift values are quoted relative to $\alpha$-Fe at 300 K.

\section{Results}

\subsection{X-ray diffraction}

X-ray diffractograms of as-cast samples show that the substitution of Fe by $\mathrm{Ga}$ shifts the position of diffraction lines corresponding to bec phase of pure $\alpha$-Fe to smaller values of $2 \theta$. Figure 1a shows, as an example, the XRD pattern of $\mathrm{Fe}_{75} \mathrm{Ga}_{25}$ alloy. The increase of the lattice parameter, $a$, caused by Ga addition can be expressed by (Fig. 1b):

$$
a=0.2869(2)+0.00020(1) y \quad[\mathrm{~nm}]
$$

where $y$ is the Ga content (at. \%) of the alloy. Additional weak reflections due to the possible occurrence of $\mathrm{DO}_{3}$ ordering of the structure are not detected. In fact, as it was said above, the similarity between the atomic scattering factors of $\mathrm{Fe}$ and $\mathrm{Ga}$ makes this detection difficult, unlike for FeSi alloys.

\subsection{Magnetic properties}

The saturation magnetization, $M_{S}$, at $300 \mathrm{~K}$, shows a linear dependence with the Ga content, $y$, up to 20 at. \% Ga (see Fig. 2a.), that can be expressed by: 
Intermetallics. Vol. 15. Núm. 2. 2007. Pag. 193-200

http://dx.doi.org/10.1016/j.intermet.2006.05.007

$$
M_{S}=223(6)-3.0(5) y \quad[\mathrm{emu} / \mathrm{g}]
$$

The average magnetic moment per Fe atom, $\left\langle\mu_{F e}\right\rangle$, decreases as the Fe content decreases (Fig. 2b). A linear dependence of $\left\langle\mu_{F e}\right\rangle$ with $y$ is observed for Ga content up to $20 \%$, that can be given by:

$$
<\mu_{F e}>=2.24(3)-0.004(3) y \quad\left[\mu_{B}\right]
$$

The different behaviour of $M_{S}$ and $<\mu_{F e}>$ below and above $y=20$ at. \% suggests a change in the structural ordering of the binary alloy.

The Curie temperature, $T_{C}$, of the arc-melted alloys decreases monotonously with the Ga content of the alloy as can be observed in Fig. 2c.

\subsection{Mössbauer spectrometry}

Fig. 3 shows Mössbauer spectra of the studied alloys recorded at room temperature. The hyperfine structure is strongly dependent on Ga content. For $y \leq 20$ at. $\%$ a six line pattern is observed, with increasing line widths as the Ga content of the alloy increases. The Mössbauer spectrum corresponding to $\mathrm{Fe}_{75} \mathrm{Ga}_{25}$ alloy is qualitatively different to those of alloys with lower Ga content, exhibiting additional and sharper lines that suggest some structural change.

The fitting of the spectra was first tried using several independent sextets with free width but only the spectrum corresponding to the alloy with 5 at.\% Ga could be satisfactorily fitted using this model. Neither the spectrum of the 25 at.\% Ga could be clearly decomposed into two components with proportions $3: 1$, as could be expected for a stoichiometric $\mathrm{Fe}_{3} \mathrm{Ga}$ with a $\mathrm{DO}_{3}$ structure.

The best fitting of the spectra was attained using a discrete magnetic hyperfine field distribution (HFD) with a linear correlation between the magnetic hyperfine field and the isomer shift of the components of the distribution in order to reproduce the 
Intermetallics. Vol. 15. Núm. 2. 2007. Pag. 193-200

http://dx.doi.org/10.1016/j.intermet.2006.05.007

asymmetrical shape of the spectra [14]. The quadrupolar splitting was averaged to zero.

The obtained HFDs are shown in Fig. 3 (right hand side).

HFDs corresponding to $y=5$ and 10 at. \% Ga range from 25 to $36 \mathrm{~T}$ and show no significant differences: only a small shift of the maximum from $32 \mathrm{~T}$ to $31.5 \mathrm{~T}$ and a small increase in the width of the distribution from $3.5 \mathrm{~T}$ to $4.6 \mathrm{~T}$ (for a Gaussian fitting) with increasing Ga content. For $y=20$ at. $\%$ Ga alloy, the HFD corresponds to a much broader six-line pattern spectrum and ranges from 18 to $36 \mathrm{~T}$ with a main maximum at $30.5 \mathrm{~T}$ and a shoulder at $21.5 \mathrm{~T}$. The magnetic hyperfine field distribution of $\mathrm{Fe}_{75} \mathrm{Ga}_{25}$ alloy, that ranges from 16 to $36 \mathrm{~T}$, consists of two clear maxima, labelled as "a" and "b" in Fig. 3, centred at $20 \mathrm{~T}$ and $30.5 \mathrm{~T}$ and with fraction areas of 0.54 and 0.46 , respectively. For a correct fitting of the spectrum it was necessary to add a number of components with magnetic hyperfine fields ranging from 1.5 to $16 \mathrm{~T}(\sim 4 \%$ of the total Fe atoms).

The average magnetic hyperfine field at $300 \mathrm{~K}$ decreases with increasing Ga content (Fig. 4a) as does the average magnetic moment per Fe atom, and a linear dependence between these two quantities is found for Ga content $\leq 20$ at. \% (Fig. 4c).

The decrease of $\left\langle B_{h \uparrow}\right\rangle$ with $y$, for $y$ up to 20 at. \%, can be expressed by a linear relation:

$$
<B_{h f}>=33.1(3)-0.23(2) y
$$

The value of $\left\langle B_{h f}>\right.$ at $y=0$ at. $\%$ of $\mathrm{Ga}$ is in agreement with the expected value of $B_{h f}$ for pure $\alpha-F e$ phase at room temperature, 33.0 T. For comparison, Fig.4a also shows the compositional dependence of $<B_{h f}>$ for FeAl [15] and FeSi [16] alloys. A discontinuous jump at 20 at.\% metalloid is encountered for FeGa and FeAl alloys, but this jump is not so clear for FeSi alloys. 
Intermetallics. Vol. 15. Núm. 2. 2007. Pag. 193-200

http://dx.doi.org/10.1016/j.intermet.2006.05.007

The average isomer shift (Fig. 4b) increases linearly with Ga content up to 20 at.\% Ga following the relation:

$$
<I S>=-0.003(5)+0.0070(5) y \quad[\mathrm{~mm} / \mathrm{s}]
$$

The angle $\theta$, defined by the direction of the magnetic hyperfine field and the propagation direction of the $\gamma$ rays, can be obtained from the intensities of the second and the third absorption lines of the spectra. For the present alloys, a value of around $55^{\circ}$ reveals the absence of texture of magnetic moments for all alloys.

\section{Local order modelling}

Using a binomial distribution, the probability, $P_{n m}$, of a bcc environment with $n$ Ga atoms as near neighbours (NN), in the first shell, and $m$ Ga atoms as next near neighbours (NNN), in the second shell is given by the expression:

$$
P_{n m}=M\left[x_{G a}^{(N N)}\right]^{n}\left[x_{G a}^{(N N N)}\right]^{m}\left[x_{F e}^{(N N)}\right]^{8-n}\left[x_{F e}^{(N N)}\right]^{6-m}
$$

where $x_{U}^{(N N)}$ and $x_{U}^{(N N N)}$ are the concentration of $\mathrm{U}$ atoms $(\mathrm{Ga}$ or $\mathrm{Fe}$ ) in the set of $8 \mathrm{NN}$ atoms of the first shell and in the set of $6 \mathrm{NNN}$ atoms of the second shell, respectively, that surrounds the central Fe atom. The multiplicity, M, can be calculated as:

$$
M=\frac{8 !}{(8-n) ! n !} \cdot \frac{6 !}{(6-m) ! m !}
$$

However, the composition of the neighbourhood of the Fe atoms must be strongly affected, not only by the global composition, but also by the ordering of the crystalline structure. In a fully disordered A2 structure, the Ga and Fe positions are indistinguishable and their distribution would be random. Therefore, the atomic composition of the first and second shells is the same, $x_{F e}$ and $x_{G a}$, for Fe and Ga atoms, respectively. However, it is important to note that using Mössbauer spectrometry (MS), from the 15 atoms (one central surrounded by 8 as $\mathrm{NN}$ and 6 as $\mathrm{NNN}$ ) which are probed 
Intermetallics. Vol. 15. Núm. 2. 2007. Pag. 193-200

http://dx.doi.org/10.1016/j.intermet.2006.05.007

and form the cluster unit, the central one is forced to be a Fe atom. Therefore, the probabilities for finding a $\mathrm{Fe}$ atom or a $\mathrm{Ga}$ atom in the neighbourhood of the probe are modified with respect to the global composition $\left(\mathrm{Fe}_{100-\mathrm{y}} \mathrm{Ga}_{\mathrm{y}}\right.$, with $x_{\mathrm{Fe}}=(100-\mathrm{y}) / 100$, $\left.x_{G a}=\mathrm{y} / 100\right)$. In fact, the average number of Fe atoms in a group of 15 is $15 x_{F e}$ and the average number of $\mathrm{Ga}$ atoms is $15 x_{G a}$. Taking into account that the central probe is a $\mathrm{Fe}$ atom, the average number of Fe atoms in the $8 \mathrm{NN}$ plus $6 \mathrm{NNN}$ will be $15 x_{F e^{-1}}$ and, thus, the corrected Fe concentration, $x_{F e}^{c o r}=\left(15 x_{F e}-1\right) / 14$, in the NN plus NNN environment is decreased with respect to the global value, and the corrected $\mathrm{Ga}$ concentration, $x_{G a}^{c o r}=15 x_{G a} / 14$, is enhanced with respect to its global concentration in the alloy.

In the case of ordered structures, the positions of $\mathrm{Ga}$ and $\mathrm{Fe}$ atoms in the elementary cell are distinguishable. For a perfect ordered $\mathrm{DO}_{3}$ structure, only possible for an alloy composition of $\mathrm{Fe}_{75} \mathrm{Ga}_{25}$, a $\mathrm{Ga}$ atom is always surrounded by $8 \mathrm{Fe}$ atoms as $\mathrm{NN}$ and $6 \mathrm{Fe}$ atoms as NNN, but for Fe atoms, two different sites are present; namely, $1 / 3$ of the Fe atoms have $8 \mathrm{Fe}$ atoms as NN and $6 \mathrm{Ga}$ atoms as NNN (D site) and 2/3 have $4 \mathrm{Fe}$ and $4 \mathrm{Ga}$ atoms as $\mathrm{NN}$ and $6 \mathrm{Fe}$ atoms as NNN (A site). (Table 1).

For alloy compositions $\mathrm{Fe}_{100-\mathrm{y}} \mathrm{Ga}_{\mathrm{y}}$ with $\mathrm{y}<25$ at. $\%$, there is no possibility for a perfect ordered structure as the number of Fe atoms is in excess and the number of $\mathrm{Ga}$ atoms is less than the needed. Therefore, we assume a "maximum ordered" structure in which Ga atoms will be always located in their correct sites and the Ga sites which can not be occupied by Ga atoms will be filled with the exceeding Fe atoms. Thus, "maximum ordered" structure implies that a Fe site will never be occupied by a Ga atom but some Ga sites will be occupied by Fe atoms, resulting three different sites for Fe (Table 1): A and D sites, which are correct Fe sites, and Ga sites occupied by Fe 
Intermetallics. Vol. 15. Núm. 2. 2007. Pag. 193-200

http://dx.doi.org/10.1016/j.intermet.2006.05.007

atoms. The distribution of the 100-y Fe atoms will be as follows: 50 will be in A sites, 25 in D sites and 25-y Fe atoms will be substituting Ga atoms.

Each $\mathrm{Fe}$ atom in an $\mathrm{A}$ site in a perfect ordered $\mathrm{DO}_{3}$ structure has $4 \mathrm{Ga}$ atoms as $\mathrm{NN}$ but for a Ga content lower than 25 at. \%, a Fe atom in an A site could have 4, 3, 2, 1 or $0 \mathrm{Ga}$ atoms as $\mathrm{NN}$. Analogously, the possible configurations for a Fe atom in a D site ranges from 6 to $0 \mathrm{Ga}$ atoms as NNN. Finally, a Fe atom in a "Ga atom-site" will always have $0 \mathrm{Ga}$ atoms as $\mathrm{NN}$ and $0 \mathrm{Ga}$ atoms as $\mathrm{NNN}$ (Table 1).

Taking into account the previous considerations to obtain the concentration of $\mathrm{Fe}$ and $\mathrm{Ga}$ atoms in the $\mathrm{NN}$ and $\mathrm{NNN}$ sets of atoms, the probabilities of the different configurations can be calculated using equation (6). The results for disordered and ordered structures are shown in figures 5 and 6 , respectively.

\section{Discussion}

(a) Alloys with low Ga content ( $\leq 10$ at. \%)

Assuming the effect of the Ga atoms on the magnetic hyperfine field to be proportional to the number of Ga atoms in the first shell, $n$, and the number of Ga atoms in the second shell, $m$, the average magnetic hyperfine field can be expressed by:

$$
<B_{h f}>=33-\sum_{n, m} P_{n m} n \cdot q-\sum_{n, m} P_{n m} m \cdot r
$$

where $q$ and $r$ are the reduction of $B_{h f}$ per Ga atom in the first and the second shell, respectively. The linearity between the reduction of $B_{h f}$ and the number of Ga atoms as $\mathrm{NN}$ (or NNN) yielding to $q$ (or $r$ ) is not a strong hypothesis for low Ga containing alloys (expected to be in a disordered structure) as the most probable configurations are those of 0,1 or $2 \mathrm{Ga}$ atoms as $\mathrm{NN}$ or $\mathrm{NNN}$ (Fig. 5). For fully disordered systems, the relationships between both $P_{n m} n$ and $P_{n m} m$ with $y$ are linear (Fig. 7). Therefore, from equations (4) and (8) it is possible to obtain: 


$$
q+0.75 r=2.3
$$

It should be remembered that expression (9) has been obtained for compositions for which the most probable environments correspond to $\mathrm{n}=0,1$ and 2 atoms of Ga.

\section{b) $\mathrm{Fe}_{75} \mathrm{Ga}_{25}$ alloy}

A perfect ordered $\mathrm{DO}_{3}$ structure (Table 1) cannot explain the fraction areas obtained for the maxima labeled as "a" and "b" of the magnetic hyperfine field distribution of $\mathrm{Fe}_{75} \mathrm{Ga}_{25}$ alloy (Fig. 3), as the probabilities for the only two possible $\mathrm{Fe}$ sites are $2 / 3$ for an $\mathrm{A}$ site and $1 / 3$ for a $\mathrm{D}$ site.

However, a simple model can explain the experimental values of the fraction areas: 0.54 for peak "a" and 0.46 for peak "b". Let us consider three types of Fe sites: $\mathrm{A}^{*}, \mathrm{D} *$ and $\mathrm{Fe}$ in $\mathrm{Ga}$ atoms sites. If $\mathrm{Ga}$ and $\mathrm{Fe}$ atoms which are not in their correct position were randomly interchanged, the ratio between the populations of $\mathrm{A}^{*}$ and $\mathrm{D}^{*}$ sites occupied by Fe atoms would remain the same as that of A and D sites in the perfect ordered structure $(2: 1)$. From the experimental fraction areas of the peaks, the only possible choice is to assign the whole peak "a", at 20 T, to A* sites (54\%) and the peak "b", at $\sim 30.5 \mathrm{~T}$, to both $\mathrm{D*}$ sites (a half of the number of Fe atoms in A sites, $27 \%$ ) and some $\mathrm{Fe}$ in $\mathrm{Ga}$ sites (the rest of $\mathrm{Fe}$ atoms, $19 \%$ ). Then, it is possible to calculate a degree of order for our $\mathrm{Fe}_{75} \mathrm{Ga}_{25}$ alloy. If a statistical set of 100 atoms is considered, 75 atoms of them will be Fe atoms, of which 40.5 will be in A sites, 20.25 in D sites and 14.25 in Ga sites; and 25 atoms will be Ga atoms, of which 14.25 will occupy Fe sites and 10.75 will remain in Ga sites. Therefore, $71.5 \%$ of the atoms are in ordered positions, which can be considered as the degree of order of the system. Although this order parameter is straightforward from the discussion above (the percentage of atoms which are in ordered positions), a comparison with standard parameters will be helpful. An extended model of the Bragg-William-Gorsky model 
Intermetallics. Vol. 15. Núm. 2. 2007. Pag. 193-200

http://dx.doi.org/10.1016/j.intermet.2006.05.007

proposed by Inden [17] yields a description of the atomic distributions in binary bcc solid solutions taking into account nearest and next nearest neighbours. The bcc lattice is divided in four fcc lattices (I, II, III and IV) with a lattice parameter which is double of that of the bcc lattice. In a perfect ordered $\mathrm{DO}_{3}$ structure, I and II lattices corresponds to the A sites of Fe, the III lattice to the Ga sites and the IV lattice to the D sites of Fe. Any atomic configuration (disordered $\mathrm{A} 2$, ordered $\mathrm{B} 2$ or ordered $\mathrm{DO}_{3}$ structures) can be described using three independent parameters [17]:

$$
\begin{aligned}
& X=\frac{1}{4}\left(p_{F e}^{I}+p_{F e}^{I I}-p_{F e}^{I I I}-p_{F e}^{I V}\right) \\
& Y=\frac{1}{2}\left(p_{F e}^{I I I}-p_{F e}^{I V}\right) \\
& Z=\frac{1}{4}\left(p_{F e}^{I}-p_{F e}^{I I}\right)
\end{aligned}
$$

where $p_{F e}^{L}$ is the occupation probability of Fe in the lattice L. Therefore, for a disordered A2 structure, $X=Y=Z=0$; for an ordered $B 2$ structure $X \neq 0$ and $Y=Z=0$ and, for a perfect ordered $\mathrm{DO}_{3}$ structure $\left(p_{F e}^{A \text { site }}=2 / 3, p_{F e}^{D \text { site }}=1 / 3\right.$ and $\left.p_{F e}^{\text {Ga site }}=0\right), \mathrm{X}=1 / 12, \mathrm{Y}=-1 / 6$ and $\mathrm{Z}=0$. In the studied case, $\mathrm{X}, \mathrm{Y}$ and $\mathrm{Z}$ would be:

$$
\begin{aligned}
& X=\frac{1}{4}\left(p_{F e}^{A \text { site }}-p_{F e}^{G a \text { site }}-p_{F e}^{D \text { site }}\right)=0.02 \\
& Y=\frac{1}{2}\left(p_{F e}^{G a \text { site }}-p_{F e}^{D \text { site }}\right)=-0.04 \\
& Z=0
\end{aligned}
$$

The actual environment of the Fe atoms in $\mathrm{Fe}_{75} \mathrm{Ga}_{25}$ alloy in this non-perfect ordered $\mathrm{DO}_{3}$ structure must be modified with respect to that of the perfect ordered one (Table 1). It is possible to estimate an average effect of the separation from the perfect $\mathrm{DO}_{3}$ ordered structure, as far as only $43 \%$ of Ga atoms and $81 \%$ of Fe atoms are in correct positions. Considering the calculated disorder and the fact that Ga atoms will occupy both $\mathrm{A}^{*}$ and $\mathrm{D} *$ sites with the same probability, the modified averaged sites will be: 
- $\quad$ Average A* site: $2.5 \mathrm{Ga}$ as NN (43\% of $4 \mathrm{Ga}$ remains in their sites and $19 \%$ of Fe are displaced) and 1.1 Ga as NNN.

- $\quad$ Average D* site: $1.5 \mathrm{Ga}$ as $\mathrm{NN}$ and $2.6 \mathrm{Ga}$ as $\mathrm{NNN}$.

- $\quad$ Average Fe site substituting a Ga site: $1.5 \mathrm{Ga}$ as NN and 1.1 Ga as NNN.

The similarity of the average neighbourhood (same first shell) of a Fe atom in a D* site and that of a Fe in a Ga site could explain the overlapping between these two contributions in the magnetic hyperfine field distribution (peak "b"). On the other hand, the non-zero average number of $\mathrm{Ga}$ as $\mathrm{NN}$ in a $\mathrm{D}^{*}$ site could help to explain the shift of peak " $b$ " with respect to $33 \mathrm{~T}$ (pure Fe), neglecting the effect of the second shell. This result, in a perfect ordered $\mathrm{DO}_{3}$ structure, could only be explained by the effect of Ga atoms as $\mathrm{NNN}$ as a $\mathrm{D}$ site has $0 \mathrm{Ga}$ as $\mathrm{NN}$.

The analysis performed above concerns only to average neighbourhood configurations. The possible deviations from the average configurations, related with a larger number of $\mathrm{Ga}$ atoms as $\mathrm{NN}$, can explain the low $B_{h f}$ values necessary to correctly fit the MS spectrum of $\mathrm{Fe}_{75} \mathrm{Ga}_{25}$ alloy.

Assuming that maxima "a" and "b" are ascribed to the A* and D* sites, respectively, it would be possible to write:

$$
\begin{gathered}
2.5 q+1.1 r=13 \\
1.5 q+2.6 r=2.5
\end{gathered}
$$

Both expressions cannot be fulfilled at the same time as this should imply a negative value of $r \sim-2.5 \mathrm{~T}$. The reason for that could be the non-linearity between $\Delta B_{h f}=33-<B_{h P}>(\mathrm{T})$ and $n$ and $m$ (i.e. $q$ and $r$ are not constant and independent of $n$ and $m$, respectively).

c) General dependence of $\left\langle B_{h f}\right\rangle$ on the number of Ga atoms as $N N$ 
Intermetallics. Vol. 15. Núm. 2. 2007. Pag. 193-200

http://dx.doi.org/10.1016/j.intermet.2006.05.007

Assuming the effect of the second shell can be negligible (i.e. the main effect on the magnetic hyperfine field is due to the first shell), it would be possible to obtain the dependence of $\left\langle B_{h f}>\right.$ on the average number of Ga atoms as NN, $<n>$, for fully disordered and "maximum ordered" structures (Fig. 8). The average values obtained for ordered and disordered structures differ only for the alloys with 20 and 25 at. \% Ga. The actual value for these alloys must lie between these two calculated values. Figure 8 also shows the experimental $B_{h f}$ values corresponding to maxima "a" and "b" of the HFD of $\mathrm{Fe}_{75} \mathrm{Ga}_{25}$ alloy as a function of their corresponding $<n>$. One single line formed by two linear branches with a slope change at $<n>\sim 2$ can be used to describe the whole set of data. Therefore, it could be concluded that $q$ is not independent of $\langle n>$. For low $<n>$ values $(\leq 2)$, the decrease of $B_{h f}$ per Ga atom as $\mathrm{NN}$ is lower than for values of the average number of $\mathrm{Ga}$ atoms as $\mathrm{NN}$ bigger than 2. This feature is also described for FeSi alloys [16]. A comparison between figure 8 and figure 4 of ref. [16] shows that, for low $<n>$ (disordered alloys, low Ga content), the effect of Ga as NN is similar to that of Si (slope $\sim 2.5 \mathrm{~T}$ per Ga or Si atom as NN), and for $<n>>2$, this effect is enhanced for both alloy types, but seems to be stronger in the case of Ga than in the case of Si (slope $\geq 15$ per Ga atom and $\sim 5.5 \mathrm{~T}$ per $\mathrm{Si}$ atom as $\mathrm{NN}$ ).

Mössbauer spectrum of $\mathrm{Fe}_{80} \mathrm{Ga}_{20}$ alloy has turned to be the most complex of the studied alloy series. Its HFD shows two broad and overlapped maxima, which can be ascribed to a mixture of the disordered $\mathrm{A} 2$ and ordered $\mathrm{DO}_{3}$ phases. The large number of relevant contributions for both fully ordered and maximum disordered approaches (see figures 5 and 6) prevents a further discussion on the hyperfine structure of this alloy. In fact, the structure of this alloy can be considered as a transition between low Ga containing alloys ( $\leq 10$ at. $\%$ ), which can be described by a disordered structure, 
Intermetallics. Vol. 15. Núm. 2. 2007. Pag. 193-200

http://dx.doi.org/10.1016/j.intermet.2006.05.007

and $\mathrm{Fe}_{75} \mathrm{Ga}_{25}$ alloy, which can be described using a $\mathrm{DO}_{3}$ ordered structure with small perturbations.

\section{Conclusions}

The substitution of Fe by 5, 10, 20 and 25 at. \% Ga in arc-melted polycrystalline FeGa binary alloys causes a decrease of the Curie temperature, the average magnetic hyperfine field, $\left\langle B_{h P}>\right.$, and the average magnetic moment, $<\mu_{\mathrm{Fe}}>$ per Fe atom at $300 \mathrm{~K}$. A linear dependence of $\left\langle B_{h f}\right\rangle$ and $\left\langle\mu_{F e}>\right.$ with Ga content, and therefore between these two quantities, is found for Ga content up to 20 at. \%.

The change in the ordering of the crystal structure with increasing Ga content of the alloy was modelled using a binomial distribution method taking into account both the nearest neighbours and the next nearest neighbours shells. Low Ga containing alloys $\left(\leq 10\right.$ at. $\%$ ) present a disordered $\mathrm{A} 2$ structure and the $\mathrm{Fe}_{80} \mathrm{Ga}_{20}$ alloy shows a mixture of both $\mathrm{A} 2$ and $\mathrm{DO}_{3}$ phases. The alloy $\mathrm{Fe}_{75} \mathrm{Ga}_{25}$ does not present a perfect ordered $\mathrm{DO}_{3}$ structure, as could be expected for its stoichiometry and a degree of order of $71.5 \%$ was calculated. The modelled actual environment of the Fe atoms in a non-perfect ordered $\mathrm{DO}_{3}$ structure (for $\mathrm{Fe}_{75} \mathrm{Ga}_{25}$ alloy) can explain the experimental features of its magnetic hyperfine field distribution using only a nearest neighbours approximation. The decrease of the magnetic hyperfine field per Ga atom in the nearest neighbours shell is $\sim 2.5 \mathrm{~T}$ for a number of $\mathrm{Ga} \leq 2$ and $\sim 5.5 \mathrm{~T}$ for higher number of $\mathrm{Ga}$ atoms.

\section{Acknowledgements}

This work was supported by the Spanish Government and EU-FEDER (Project MAT2004-04618) and by the PAI of the Junta de Andalucía. One of the authors (J.S.B.) acknowledges a research contract by the regional Government of Andalucia. 


\section{References}

[1] A.E. Clark, J.B. Restorff, M. Wun-Fogle, T.A. Lograsso and D.L. Schlagel, IEEE Trans. Magn. 36 (2000) 3238.

[2] N. Srisukhumbowornchai and S. Guruswany, J. Appl. Phys. 90 (2001) 5680.

[3] A.E. Clark, K.B. Hathaway, M. Wun-Fogle, J.B. Restorff, T.A. Lograsso, V.M. Keppens, G. Petculescu and R.A. Taylor, J. Appl. Phys. 93 (2003) 8621.

[4] C. Bormio-Nunes, M.A. Tirelli, R. Sato Turtelli, R. Grössinger, H. Müller, G. Wiesinger, H. Sassik and M. Reissner, J. Appl. Phys. 97 (2005) 033901.

[5] S. Guruswany, N. Srisukhumbowornchai, A.E. Clark, J.B. Restorff and M. WunFogle, Scr. Mater. 43 (2000) 239.

[6 ] A.E. Clark, M. Wun-Fogle, J.B. Restorff, T.A. Lograsso and J.R. Cullen, IEEE Trans. Magn. 37 (2001) 2678.

[7] N. Srisukhumbowornchai and S. Guruswany, J. Appl. Phys. 92 (2002) 5371.

[8] O. Ikeda, R. Kainuma, I. Ohnuma, K. Fukamichi and K. Ishida, J. Alloy Compd. 347 (2002) 198.

[9] T.A. Lograsso, A.R. Ross, D.L. Schlagel, A.E. Clark and M. Wun-Fogle, J. Alloy Compd. 350 (2003) 95.

[10] J.M. Borrego, C.F. Conde, A. Conde, M. Stoica, S. Roth and J.M. Greneche, to be published.

[11] J. Restrepo and G.A. Perez Alcázar, phys. stat. sol. (b) 220 (2000) 395.

[12] R.A. Brand, J. Lauer, and D.M. Herlach, J. Phys. F: Met. Phys. 12 (1983) 675.

[13] J. Teillet and F. Varret, MOSFIT program (Université du Maine, Le Mans) unpublished.

[14] I. Vincze, Solid State Commun. 25 (1978) 689. 
Intermetallics. Vol. 15. Núm. 2. 2007. Pag. 193-200

http://dx.doi.org/10.1016/j.intermet.2006.05.007

[15] D.S. Schmool, E. Araujo, M.M. Amado, M. Alegria Feio, D. Martín Rodríguez, J.S. Garitaonandia and F. Plazaola, J. Magn. Mag. Mater. 272-276 (2004) 1342.

[16] M.B. Stearns, Phys. Rev. 129 (1963) 1136.

[17] G. Inden and W. Pitsch, Z. Metallkde, 62 (1971) 627. 
Intermetallics. Vol. 15. Núm. 2. 2007. Pag. 193-200

http://dx.doi.org/10.1016/j.intermet.2006.05.007

Table 1. Definition of the different Fe sites in a perfect ordered $\mathrm{DO}_{3}$ structure and in a “maximum ordered" structure for $\mathrm{Fe}_{100-\mathrm{y}} \mathrm{Ga}_{\mathrm{y}}$ alloys.

\begin{tabular}{|c|c|c|c|c|c|}
\hline Structure & Site & $\begin{array}{l}\mathrm{NN} \mathrm{Fe} \\
\text { atoms }\end{array}$ & $\begin{array}{l}\mathrm{NN} \mathrm{Ga} \\
\text { atoms } \\
(\mathrm{n})\end{array}$ & $\begin{array}{c}\text { NNN Fe } \\
\text { atoms }\end{array}$ & $\begin{array}{c}\text { NNN Ga } \\
\text { atoms } \\
(\mathrm{m})\end{array}$ \\
\hline \multirow{3}{*}{$\begin{array}{c}\text { Perfect } \\
\text { ordered } \mathrm{DO}_{3} \\
\mathrm{y}=25 \text { at. } \%\end{array}$} & $\begin{array}{l}\text { Fe atom in } \\
\text { D site }\end{array}$ & 8 & 0 & 0 & 6 \\
\hline & $\begin{array}{l}\text { Fe atom in } \\
\text { A site }\end{array}$ & 4 & 4 & 6 & 0 \\
\hline & $\begin{array}{l}\text { Ga atom } \\
\text { site }\end{array}$ & 8 & 0 & 6 & 0 \\
\hline \multirow{3}{*}{$\begin{array}{l}\text { "Maximum } \\
\text { ordered" } \\
\mathrm{y}<25 \text { at. } \%\end{array}$} & $\begin{array}{l}\text { Fe atom in } \\
\text { D site }\end{array}$ & 8 & 0 & $\geq 6$ & $\leq 6$ \\
\hline & $\begin{array}{l}\text { Fe atom in } \\
\text { A site }\end{array}$ & $\geq 4$ & $\leq 4$ & 6 & 0 \\
\hline & $\begin{array}{c}\text { Fe atom } \\
\text { in a Ga atom } \\
\text { site }\end{array}$ & 8 & 0 & 6 & 0 \\
\hline
\end{tabular}


Intermetallics. Vol. 15. Núm. 2. 2007. Pag. 193-200

http://dx.doi.org/10.1016/j.intermet.2006.05.007

\section{Figure captions}

FIG. 1. (a) XRD pattern corresponding to $\mathrm{Fe}_{75} \mathrm{Ga}_{25}$ alloy and (b) lattice parameter, $a$, of the bcc phase of FeGa alloys as a function of the Ga content of the alloy.

FIG.2. (a) Compositional dependence of the saturation magnetization, $M_{S}$; (b) average magnetic moment per Fe atom, $\left\langle\mu_{F e}>\right.$, at $300 \mathrm{~K}$ and (c) Curie temperature, $T_{C}$, of $\mathrm{Fe}_{100-\mathrm{y}} \mathrm{Ga}_{\mathrm{y}}$ alloys.

FIG. 3. Transmission Mössbauer spectra at $300 \mathrm{~K}$ and their corresponding fitting (solid line) and magnetic hyperfine field distributions of $\mathrm{Fe}_{100-\mathrm{y}} \mathrm{Ga}_{\mathrm{y}}$ alloys.

FIG. 4. (a) Compositional dependence of the average magnetic hyperfine field, $<B_{h f}>$; (b) average isomer shift, $\langle I S>$, of FeGa alloys. For comparison, it is also shown the compositional dependence of $\left\langle B_{h \rho} \ngtr\right.$ for FeAl [12] and FeSi [13] binary alloys on (a). (c) Average magnetic hyperfine field, $\left\langle B_{h} \ngtr\right.$, as a function of the average magnetic moment per Fe atom, $<\mu_{F e}>$, at $300 \mathrm{~K}$.

FIG. 5. Probabilities, $P_{n m}$, of a Fe site to have $n$ Ga atoms as NN, in the first shell, and $m$ Ga atoms as NNN, in the second shell for a fully disordered $\mathrm{A}_{2}$ structure, calculated using equation (6), for the different studied alloys.

FIG. 6. Probabilities, $P_{n m}$, of a Fe site to have $n$ Ga atoms as NN, in the first shell, and $m$ Ga atoms as NNN, in the second shell for a "maximum ordered" $\mathrm{DO}_{3}$ structure, calculated using equation (6), for the different studied alloys.

FIG. 7. Summatories of the products $P_{n m} \bullet n$ and $P_{n m} \bullet m$, as a function of the Ga content for $\mathrm{Fe}_{100-\mathrm{y}} \mathrm{Ga}_{\mathrm{y}}$ alloys.

FIG. 8. $<B_{h f}>$ dependence on the average number of Ga atoms as near neighbours, $\langle n>$, for both fully disordered and "maximum ordered" structures. The experimental $B_{h f}$ 
Intermetallics. Vol. 15. Núm. 2. 2007. Pag. 193-200

http://dx.doi.org/10.1016/j.intermet.2006.05.007

values corresponding to maxima "a" and "b" of the HFD of $\mathrm{Fe}_{75} \mathrm{Ga}_{25}$ alloy are also shown. 
Intermetallics. Vol. 15. Núm. 2. 2007. Pag. 193-200

http://dx.doi.org/10.1016/j.intermet.2006.05.007

FIGURE 1
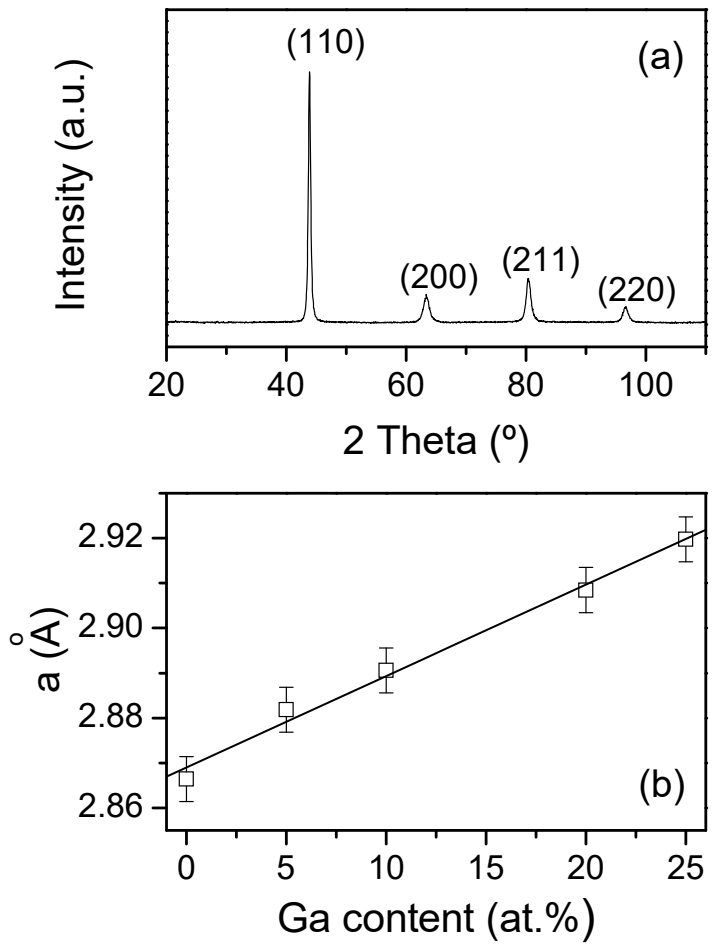
Intermetallics. Vol. 15. Núm. 2. 2007. Pag. 193-200

http://dx.doi.org/10.1016/j.intermet.2006.05.007

FIGURE 2
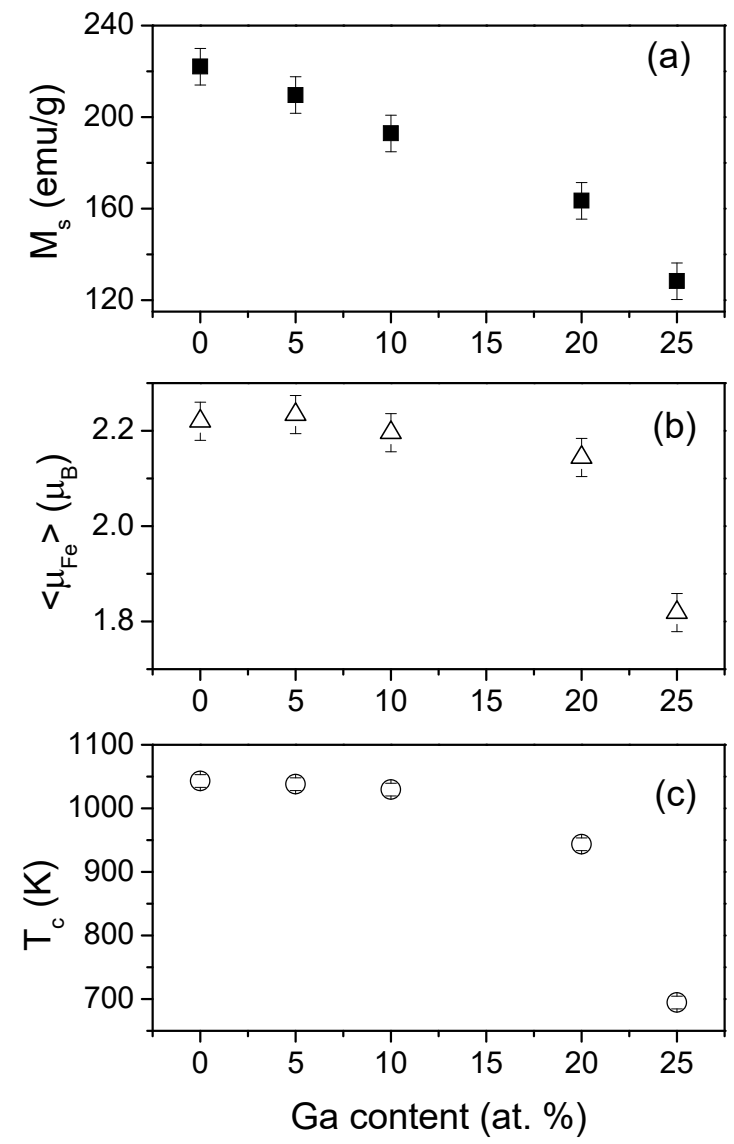
Intermetallics. Vol. 15. Núm. 2. 2007. Pag. 193-200

http://dx.doi.org/10.1016/j.intermet.2006.05.007

FIGURE 3
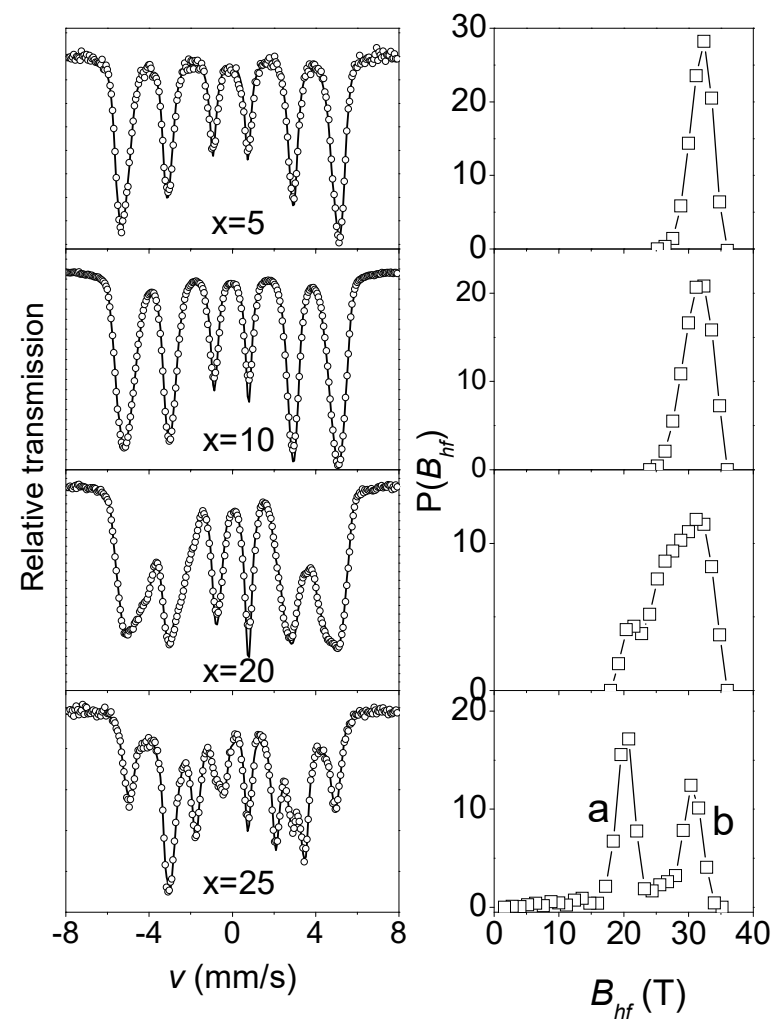
Intermetallics. Vol. 15. Núm. 2. 2007. Pag. 193-200

http://dx.doi.org/10.1016/j.intermet.2006.05.007

FIGURE 4
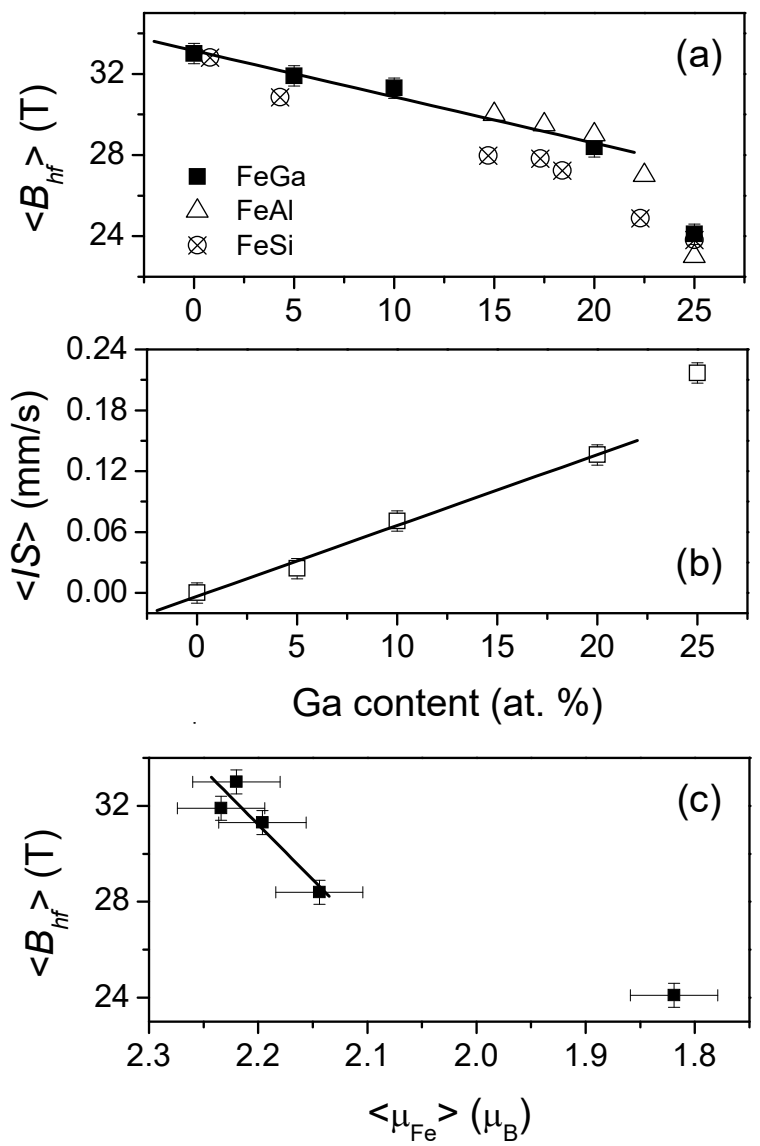
Intermetallics. Vol. 15. Núm. 2. 2007. Pag. 193-200

http://dx.doi.org/10.1016/j.intermet.2006.05.007

FIGURE 5

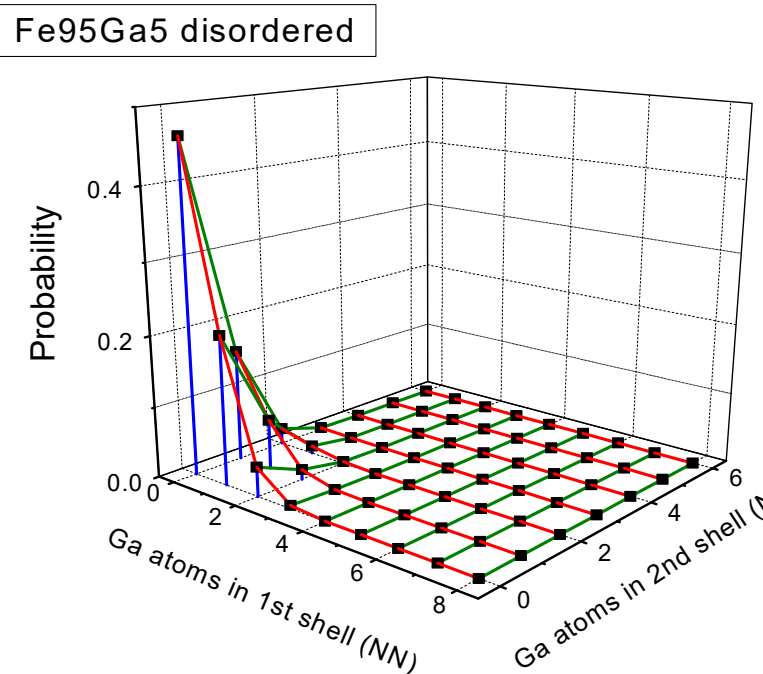

\section{Fe90Ga10 disordered}

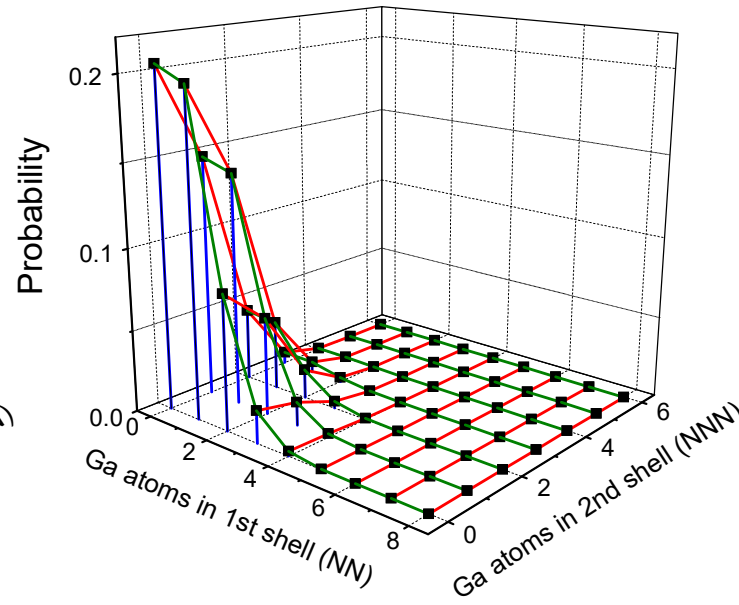

Fe80Ga20 disordered

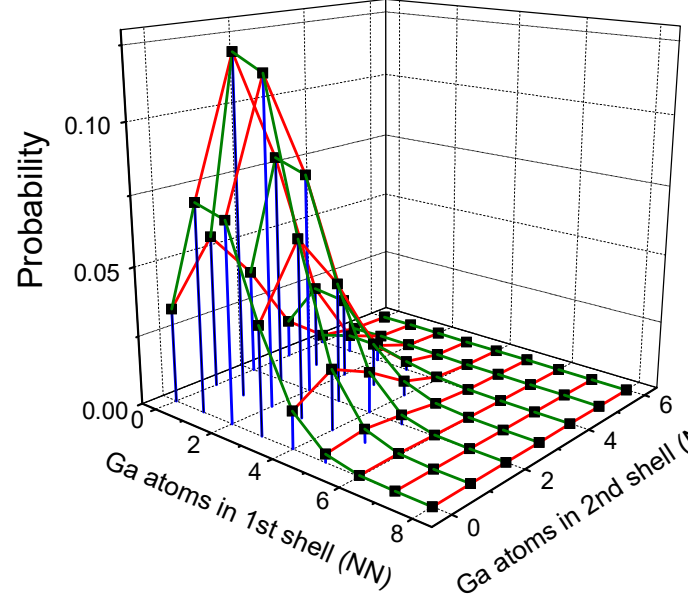

Fe75Ga25 disordered

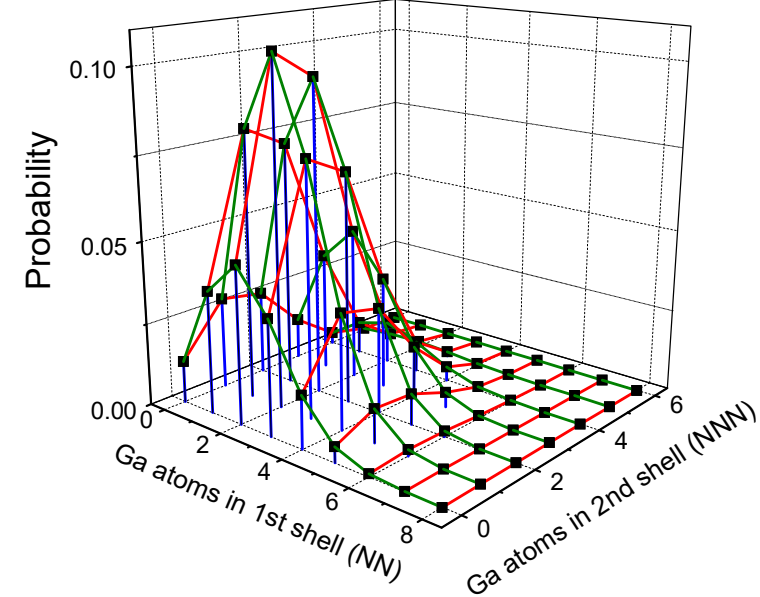


Intermetallics. Vol. 15. Núm. 2. 2007. Pag. 193-200

http://dx.doi.org/10.1016/j.intermet.2006.05.007

FIGURE 6

\section{Fe95Ga5 ordered}

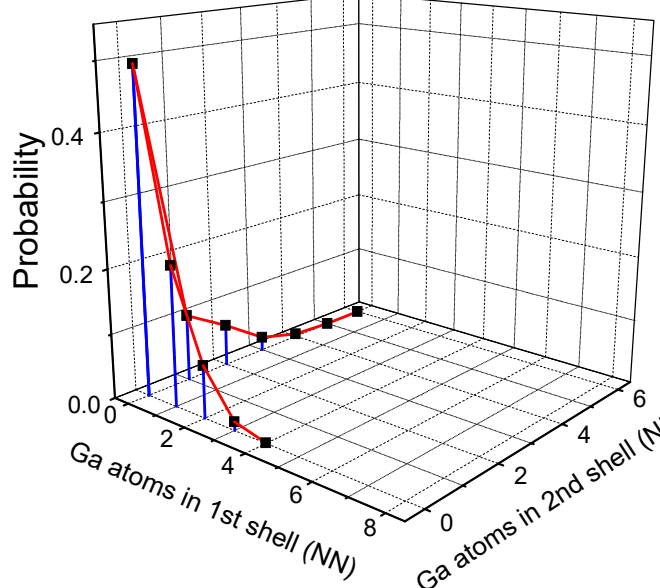

Fe80Ga20 ordered

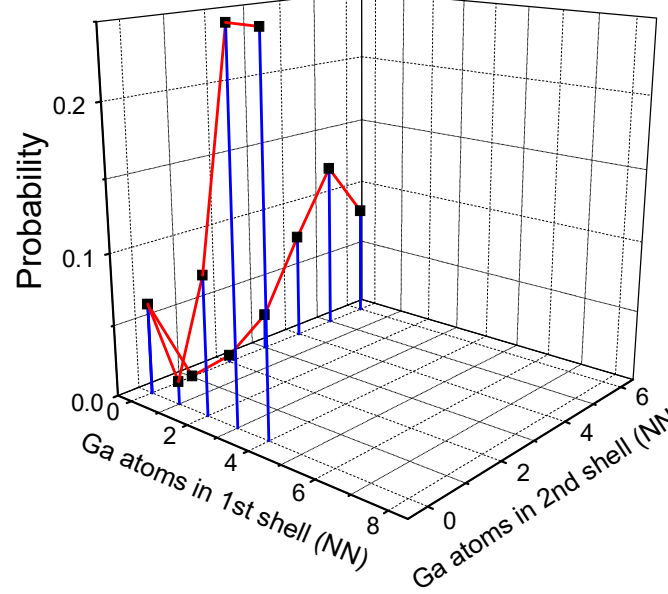

\section{Fe90Ga10 ordered}

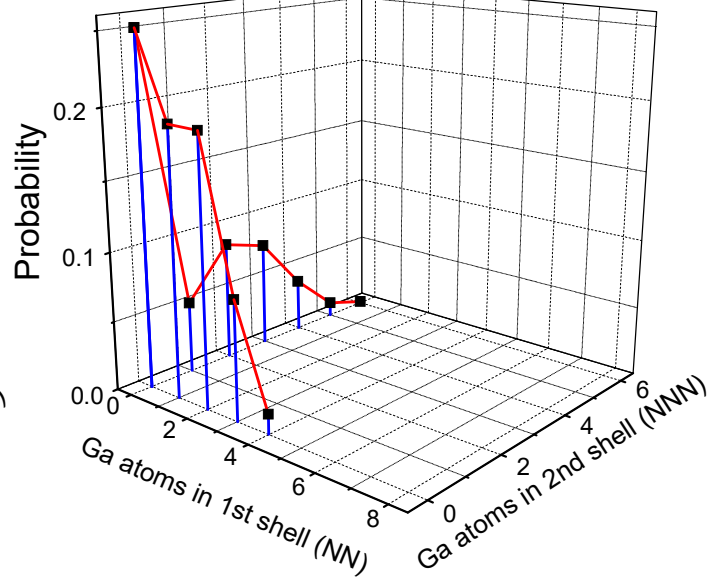

Fe75Ga25 ordered

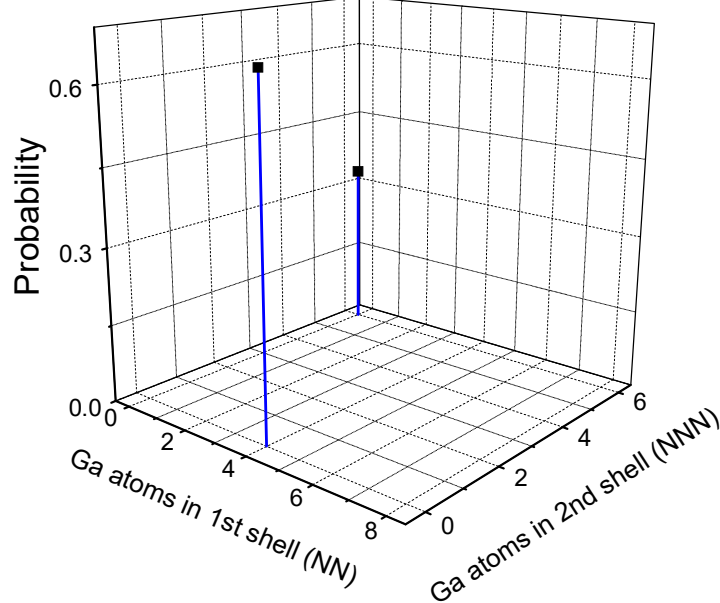


Intermetallics. Vol. 15. Núm. 2. 2007. Pag. 193-200

http://dx.doi.org/10.1016/j.intermet.2006.05.007

FIGURE 7

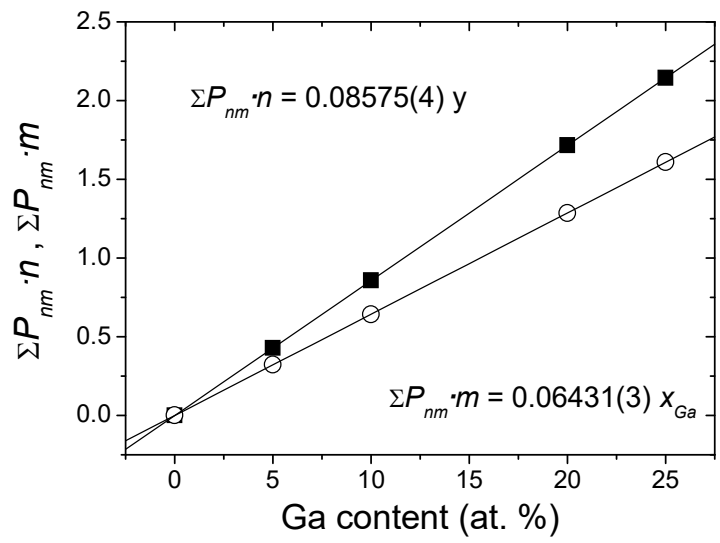


Intermetallics. Vol. 15. Núm. 2. 2007. Pag. 193-200

http://dx.doi.org/10.1016/j.intermet.2006.05.007

FIGURE 8

+ Full disorder

O Maximum order

$\Delta$ Experimental maxima "a" and "b" $\left(\mathrm{Fe}_{75} \mathrm{Ga}_{25}\right)$

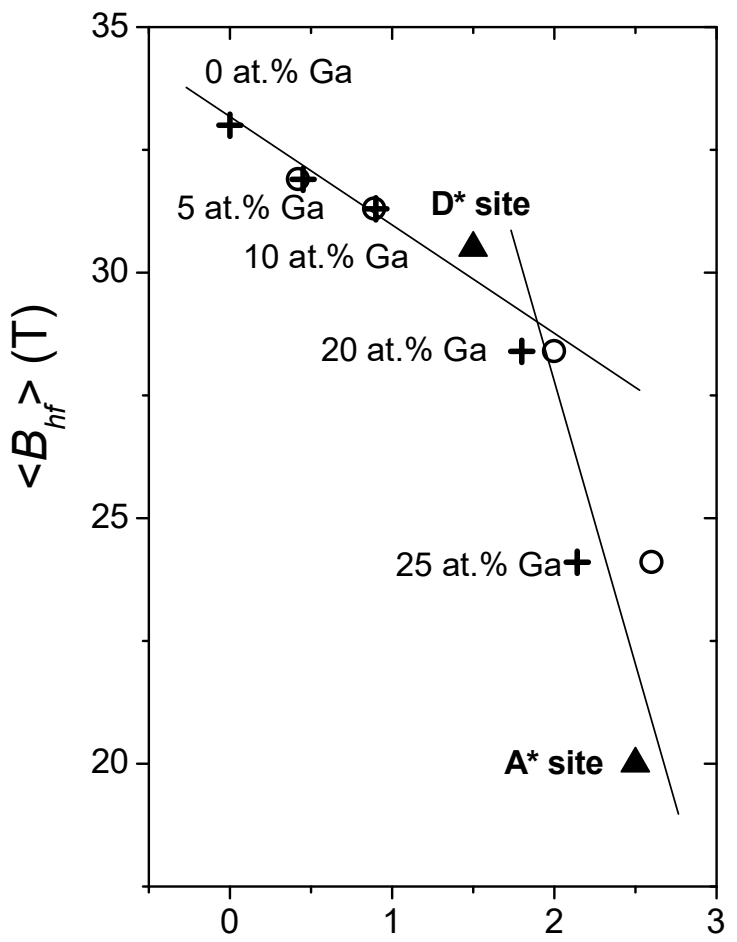

Average $\mathrm{Ga}$ atoms as $\mathrm{NN},<n>$ 